\title{
AMPLITUDE AND GAIN ERROR INFLUENCE ON TIME ERROR ESTIMATION ALGORITHM FOR TIME INTERLEAVED A/D CONVERTER SYSTEM
}

\author{
J. Elbornsson, F. Gustafsson \\ Linköping University \\ Department of Electrical Engineering \\ \{jonas,fredrik\}@isy.liu.se
}

\author{
J.-E. Eklund \\ Ericsson Microelectronics AB \\ jan-erik.eklund@mic.ericsson.se
}

\begin{abstract}
A method for blind estimation of static time errors in time interleaved A/D converters is investigated. The method assumes that amplitude and gain errors are removed before the time error estimation. Even if the amplitude and gain errors are estimated and removed, there will be small errors left. In this paper, we investigate how the amplitude and gain errors influence the time error estimation performance.
\end{abstract}

\section{INTRODUCTION}

Many digital signal processing applications, such as radio base stations or VDSL modems, require A/D converters with very high sample rate and very high accuracy. To achieve high enough sample rates, an array of $\mathrm{M} \mathrm{A/D}$ converters, interleaved in time, can be used. Each ADC should work at $1 / M$ th of the desired sample rate [1], see Figure 1. Three kinds of mismatch errors are introduced by the interleaved structure:

\section{- Time errors (static jitter)}

The delay time of the clock to the different A/D converters is not equal. This means that the signal will be periodically but non-uniformly sampled.

\section{- Amplitude offset errors}

The ground level can be slightly different in the different A/D converters. This means that there is a constant amplitude offset in each A/D converter.

\section{- Gain error}

The gain, from analog input to digital output, can be different for the different $\mathrm{A} / \mathrm{D}$ converters.

The errors are assumed to be static, so that the error is the same in the same AD-converter from one cycle to the next. There are also random errors in time, amplitude and gain due to thermal noise, which are different from one sample to the next. These errors do not have anything to do with the parallel structure of the A/Dconverter and are impossible to estimate because of their random behavior. These errors are not discussed further here.

We will in this paper focus on the time error estimation. Methods for estimation of timing errors have been presented in for instance [2] and [3] but those methods require a known calibration signal. Calibration of A/D converters is time-consuming and expensive. Therefore a lot of costs can be saved if the errors in the ADC can be automatically estimated and compensated for under drift. We will in this paper investigate a blind estimation method for timing errors in interleaved ADCs, previously presented in [4].

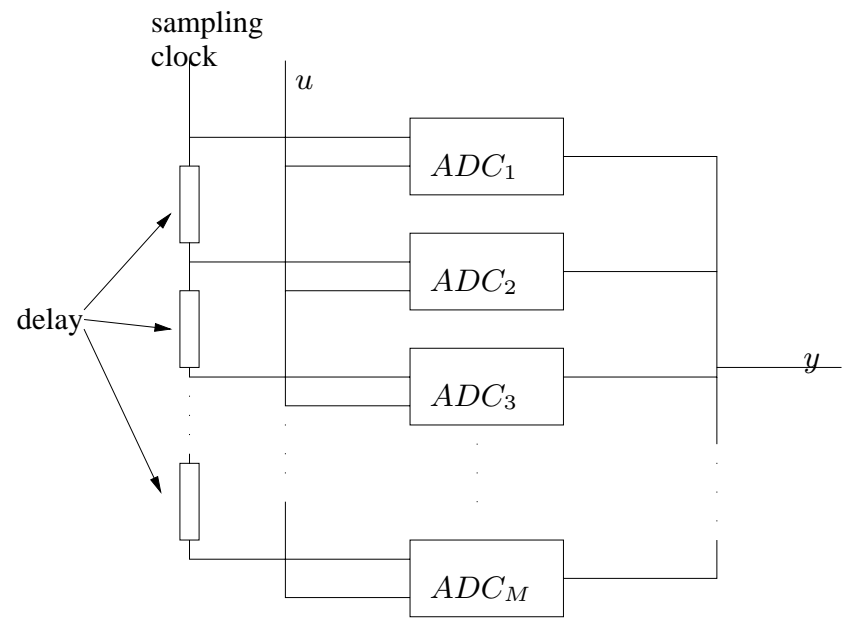

Fig. 1. M parallel ADC's with the same master clock.

This method assumes that the amplitude and gain errors are compensated for before the time error estimation. But even if this is done, there will be small amplitude and gain errors left. We will in this paper investigate how these errors influence the performance of the time error estimation algorithm.

\section{NOTATION}

The analog input signal is denoted $u(t) . T_{s}$ denotes the nominal sampling time, that we would have without any errors. $M$ is the number of $\mathrm{A} / \mathrm{D}$ converters in the parallel structure. The time offset for the $i$ th $\mathrm{A} / \mathrm{D}$ converter is denoted $t_{i}$. The output from the $i$ th $\mathrm{A} / \mathrm{D}$ converter is denoted $y_{i}[k]$ where $k$ is the $k$ th sample from that $\mathrm{A} / \mathrm{D}$ converter. Each $\mathrm{A} / \mathrm{D}$ converter form a subsequence,

$$
y_{i}[k]=\left(1+g_{i}\right) u\left((k M+i) T_{s}+t_{i}\right)+A_{i}
$$

The sample time for each such subsequence is exactly $M T_{s}$. These subsequences are merged to the output signal

$$
y[m]=y_{(m \bmod M)}\left[\left\lfloor\frac{m}{M}\right\rfloor\right]
$$

where $\lfloor\cdot\rfloor$ denotes integer part. The difference between samples from A/D converter $i-1$ and A/D converter $i$ is denoted $\Delta y_{i}[k]=$ $y_{i}[k]-y_{i-1}[k]$. We denote by $N$ the number of data points from 
each A/D converter. We assume that the same number of samples is taken from all the A/D converters so that $N M$ is the total number of data. We use the notation

$$
\bar{E}(s(t))=\lim _{n \rightarrow \infty} \frac{1}{n} \sum_{t=1}^{n} E(s(t))
$$

for quasistationary signals [5], where the expectation is taken over possible stochastic parts of $s(t)$.

\section{SIGNAL RECONSTRUCTION}

If all the error parameters are known, and the input signal $u(t)$ is band limited, $u(t)$ can be exactly reconstructed from the sampled signal $y[k]$. We will in this section describe how the different errors can be removed.

- First, the amplitude errors should be removed. This is done by subtracting the amplitude errors from the subsequences.

$$
\begin{aligned}
& z_{i}[k]=y_{i}[k]-A_{i} \\
& =\left(1+g_{i}\right) u\left((k M+i) T_{s}+t_{i}\right)
\end{aligned}
$$

- Next, the gain errors should be removed. This is done by dividing the subsequences by the correct gain.

$$
x_{i}[k]=\frac{z_{i}[k]}{1+g_{i}}=u\left((k M+i) T_{s}+t_{i}\right)
$$

- Finally, the time errors should be removed. This is done in the frequency domain [6]. Calculate the DFTs of the M subsequences $x_{i}[k], i=1, \ldots, M$ :

$$
X_{i}[n]=\operatorname{DFT}\left\{x_{i}[k]\right\}
$$

The DFT of $u_{i}[k]$ can then be calculated from $X_{i}[n]$ as

$$
\begin{aligned}
& U_{i}[n]=e^{-j \frac{2 \pi n t_{i}}{M N}} Y_{i}[n] \\
& n=-N / 2, \ldots, N / 2-1
\end{aligned}
$$

$U[n]$ can then be calculated from these $M$ subsequences.

$$
\begin{aligned}
& U[n]=\sum_{i=1}^{M} e^{-j \frac{2 \pi(i-1) n}{M N}} U_{i}[(n \bmod N)-N / 2] \\
& n=-N M / 2, \ldots, N M / 2-1
\end{aligned}
$$

The estimated uniformly sampled signal is then calculated as

$$
u[k]=I D F T\{U[n]\}
$$

If $u(t)$ is band limited to below the Nyquist frequency, $u(t)$ can be exactly reconstructed from $u[k]$.

\section{TIME ERROR ESTIMATION}

The time error estimation algorithm is here briefly reviewed. The algorithm is presented in more detail in $[4,7]$.

The algorithm is based on the assumption that the signal changes more on average if it is a long time between the samples than if it is a short time between them. Therefore we have to assume that the signal varies slowly enough, i.e. has low enough bandwidth.
We look at the difference, $\Delta y_{i}[k]$, between two adjacent samples and make a Taylor expansion around the nominal sampling time of A/D converter $i-1$.

$$
\begin{aligned}
\Delta y_{i}[k] & =y_{i}[k]-y_{i-1}[k] \\
& \approx\left(T_{s}+t_{i}-t_{i-1}\right) u^{\prime}\left(k M T_{s}+(i-1) T_{s}\right)
\end{aligned}
$$

We calculate the mean squared difference between two adjacent A/D converters:

$$
\begin{aligned}
\hat{R}_{i, i-1}^{N}[0] & =\frac{1}{N} \sum_{k=1}^{N}\left\{\Delta y_{i}[k]\right\}^{2} \\
& \rightarrow\left(T_{s}+t_{i}-t_{i-1}\right)^{2} \bar{E}\left\{\left(u^{\prime}(t)\right)^{2}\right\}, N \rightarrow \infty
\end{aligned}
$$

To estimate $\bar{E}\left(u^{\prime}(t)\right)^{2}$, an average over all the A/D-converters is calculated:

$$
\begin{aligned}
& \frac{1}{M} \sum_{i=1}^{M} \hat{R}_{i, i-1}^{N}[0] \\
& \approx T_{s}^{2}\left(1+\frac{2}{M} \sum_{i=1}^{M}\left(\frac{t_{i}^{(l)}}{T_{s}}\right)^{2}-\frac{2}{M} \sum_{i=1}^{M} \frac{t_{i}^{(l)}}{T_{s}} \frac{t_{i-1}^{(l)}}{T_{s}}\right) E\left\{\left(u^{\prime}(t)\right)^{2}\right\} \\
& =T_{s}^{2}(1+\Delta) \bar{E}\left\{\left(u^{\prime}(t)\right)^{2}\right\}
\end{aligned}
$$

Assuming that $\Delta=\frac{2}{M} \sum_{i=1}^{M} t_{i}^{2}-\frac{2}{M} \sum_{i=1}^{M} t_{i} t_{i-1}$ is small compared to $T_{s}^{2}$ we can calculate a crude estimate of the time error from the equations (10) and (11), using the first ADC as reference, i.e., $t_{0}=0$.

$$
\begin{aligned}
& t_{i}^{(0)}=T_{s} \sum_{j=2}^{i}\left(\sqrt{\frac{\hat{R}_{j, j-1}^{N}[0]}{\frac{1}{M} \sum_{i=1}^{M} \hat{R}_{i, i-1}^{N}[0]}}-1\right) \\
& i=2, \ldots, M
\end{aligned}
$$

With this estimate of the time offsets we can improve the estimate of $E\left\{\left(u^{\prime}(t)\right)^{2}\right\}$ using equation (11). Then the time error estimates can be improved by fixed-point iteration [8]:

$$
\begin{aligned}
& \text { for } i=2, \ldots, \mathrm{M} \\
& t_{i}^{(l)}=T_{s} \sum_{j=2}^{i}\left(\sqrt{\frac{1}{\frac{\hat{R}_{j, j-1}^{N}[0]}{M\left(1+\Delta^{(l-1)}\right)} \sum_{i=1}^{M} \hat{R}_{i, i-1}^{N}[0]}}-1\right) \\
& \Delta^{(l)}=\frac{2}{M} \sum_{i=1}^{M}\left(\frac{t_{i}^{(l)}}{T_{s}}\right)^{2}-\frac{2}{M} \sum_{i=1}^{M} \frac{t_{i}^{(l)}}{T_{s}} \frac{t_{i-1}^{(l)}}{T_{s}}
\end{aligned}
$$

The iteration is continued until the changes in $t_{i}^{(l)}$ are small enough. Simulations show that one or two iterations are enough.

\section{GAIN AND AMPLITUDE ERROR INFLUENCE ON TIME ERROR ESTIMATION}

We will in this section calculate the error of the time error estimate caused by remaining amplitude and gain errors. We will throughout this section assume that $M=2$ to avoid too messy expressions. We will in this section use superscript 0 to denote the estimates without gain or amplitude errors, for instance $\left(t_{i}\right)^{0}$ denotes the estimate as calculated in Section 4. 


\subsection{Gain error influence}

We use the first A/D converter as reference, i.e., we assume that the gain of the first $\mathrm{ADC}$ is 1 . We have then the two subsequences

$$
\begin{aligned}
& y_{0}[k]=u\left(2 k T_{s}\right) \\
& y_{1}[k]=\left(1+g_{1}\right) u\left(2 k T_{s}+T_{s}+t_{1}\right)
\end{aligned}
$$

A Taylor expansion of the difference between two adjacent samples can then be calculated for the two cases

$$
\begin{aligned}
\Delta y_{1}[k] & \approx g_{1} u\left(2 k T_{s}\right)+\left(1+g_{1}\right)\left(T_{s}+t_{1}\right) u^{\prime}\left(2 k T_{s}\right) \\
\Delta y_{0}[k] & \approx-g_{1} u\left(2 k T_{s}\right) \\
& +\left(T_{s}-t_{1}-T_{1} g_{1}\right) u^{\prime}\left(2(k-1) T_{s}+T_{s}\right)
\end{aligned}
$$

Following the calculations from section 4 we get the initial time error estimate as

$$
\begin{aligned}
& t_{1}^{(0)}=T_{s}\left(\sqrt{\frac{(g+1)^{2}\left\{\hat{R}_{1,0}^{N}[0]\right\}^{0}+g_{1}^{2} \bar{E}\left(u^{2}\right)}{T_{s}^{2} g_{1}^{2} \bar{E}\left(u^{2}\right)+X \frac{1}{2} \sum_{i=0}^{1}\left\{\hat{R}_{i, i-1}^{N}[0]\right\}^{0}}}-1\right) \\
& X=1+\frac{\left(1+g_{1}\right)^{2}\left(\left(T_{s}+t_{1}\right)^{2}+\left(T_{s}-t_{1}+g_{1} t_{1}\right)^{2}\right)}{2\left(T_{s}^{2}+t_{1}^{2}\right)}
\end{aligned}
$$

If we make a Taylor expansion of equation (18) around $g_{1}=0$, and assume that $t_{1}$ is small compared to $T_{s}$, we can calculate an approximative expression for the estimation error caused by gain error.

$$
\left|\frac{t_{1}^{(0)}-\left\{t_{1}^{(0)}\right\}^{0}}{\left\{t_{1}^{(0)}\right\}^{0}}\right| \approx \frac{\bar{E}\left(u^{2}\right)}{\frac{1}{2} \sum_{i=0}^{1}\left\{\hat{R}_{i, i-1}^{N}[0]\right\}^{0}} g_{1}^{2}
$$

This expression shows that the time error estimation algorithm is more sensitive to gain errors for a slowly varying input signal than for a fast varying input signal. This result is in accordance with what we would expect from (16) and (17), where we can see that $\Delta y_{i}[k]$ is more affected by gain errors if the signal is slowly varying ( $u^{\prime}$ is smaller compared to $u$ on avarage for a slowly varying signal).

\subsection{Amplitude error influence}

We assume that the amplitude error of the first A/D converter is zero, i.e., we use the amplitude offset of the first ADC as reference. This means that we have the too subsequences

$$
\begin{aligned}
& y_{0}[k]=u\left(2 k T_{s}\right) \\
& y_{1}[k]=u\left(2 k T_{s}+T_{s}+t_{1}\right)+A_{1}
\end{aligned}
$$

A Taylor expansion of the difference between two adjacent samples can then be calculated for the two cases

$$
\begin{array}{r}
\Delta y_{1}[k] \approx A_{1}+\left(T_{s}+t_{1}\right) u^{\prime}\left(2 k T_{s}\right) \\
\Delta y_{0}[k] \approx-A_{1}+\left(T_{s}-t_{1}\right) u^{\prime}\left(2(k-1) T_{s}+T_{s}\right)
\end{array}
$$

Following the calculations from section 4 we get the initial time error estimate as

$$
t_{1}^{(0)}=T_{s}\left(\sqrt{\frac{A_{1}^{2}+\left\{\hat{R}_{1,0}^{N}[0]\right\}^{0}}{A^{2}+\frac{1}{2} \sum_{i=0}^{1}\left\{\hat{R}_{i, i-1}^{N}[0]\right\}^{0}}}-1\right)
$$

The error in this estimate is

$$
\begin{aligned}
& \frac{t_{1}^{(0)}-\left\{t_{1}^{(0)}\right\}^{0}}{\left\{t_{1}^{(0)}\right\}^{0}} \\
& =\frac{\left(T_{s}+\left\{t_{1}^{(0)}\right\}^{0}\right)}{\left\{t_{1}^{(0)}\right\}^{0}}\left(\sqrt{\frac{1+\frac{A_{1}^{2}}{\left\{\hat{R}_{1,0}^{N}[0]\right\}^{0}}}{1+\frac{A_{1}^{2}}{\frac{1}{2} \sum_{i=0}^{1}\left\{\hat{R}_{i, i-1}^{N}[0]\right\}^{0}}}}-1\right)
\end{aligned}
$$

A first order Taylor expansion of the error with respect to $A^{2}$ gives

$$
\begin{aligned}
& \left|\frac{t_{1}^{(0)}-\left\{t_{1}^{(0)}\right\}^{0}}{\left\{t_{1}^{(0)}\right\}^{0}}\right| \\
& \approx\left|\frac{\left(T_{s}+\left\{t_{1}^{(0)}\right\}^{0}\right)}{\left\{t_{1}^{(0)}\right\}^{0}} \frac{A_{1}^{2}}{2} \frac{\sum_{i=0}^{1}\left\{\hat{R}_{i, i-1}^{N}[0]\right\}^{0}-2\left\{\hat{R}_{1,0}^{N}[0]\right\}^{0}}{\sum_{i=0}^{1}\left\{\hat{R}_{i, i-1}^{N}[0]\right\}^{0}\left\{\hat{R}_{1,0}^{N}[0]\right\}^{0}}\right| \\
& \approx \frac{A_{1}^{2}}{\sum_{i=0}^{1}\left\{\hat{R}_{i, i-1}^{N}[0]\right\}^{0}}
\end{aligned}
$$

The second approximation is good if the time errors are small compared to the sample interval, $T_{s}$. Equation (26) shows that the time estimation accuracy is basically affected in the same way with amplitude offset errors as for gain errors. This agrees with what we would expect from (22) and (23), where we can see that $\Delta y_{i}[k]$ is more affected by amplitude errors for a slowly varying signal than for a fast varying signal ( $u^{\prime}$ is smaller compared to $A_{1}$ on avarage for a slowly varying signal).

\subsection{Gain and amplitude error influence}

Here we study the influence of both amplitude and gain errors on the time error estimation algorithm. Using the first ADC as reference, we have the two subsequences

$$
\begin{aligned}
& y_{0}[k]=u\left(2 k T_{s}\right) \\
& y_{1}[k]=\left(1+g_{1}\right) u\left(2 k T_{s}+T_{s}+t_{1}\right)+A_{1}
\end{aligned}
$$

Following the same calculations as in Section 5.1 and 5.2 we find that the crossterms between $g_{1}$ and $A_{1}$ only occur for higher order than two. The second order terms are added constructively, which means that the errors calculated in equation (19) and equation (26) are added.

$$
\begin{aligned}
& \left|\frac{t_{1}^{(0)}-\left\{t_{1}^{(0)}\right\}^{0}}{\left\{t_{1}^{(0)}\right\}^{0}}\right| \\
& \approx \frac{\bar{E}\left(u^{2}\right)}{\frac{1}{2} \sum_{i=0}^{1}\left\{\hat{R}_{i, i-1}^{N}[0]\right\}^{0}} g_{1}^{2}+\frac{1}{\sum_{i=0}^{1}\left\{\hat{R}_{i, i-1}^{N}[0]\right\}^{0}} A_{1}^{2}
\end{aligned}
$$

\section{SIMULATIONS}

To verify the expressions calculated in Section 5 we have done some simulations with amplitude and gain errors. In the simulations, different input signals and different gain and amplitude errors have been used:

\section{- Input signals:}

Four different signals are used, three sinusoidal signals at different frequencies $(\omega \in\{0.01,0.1,1\})$ and a multi-sine signal (sum of 20 sinusoids at different frequencies). 


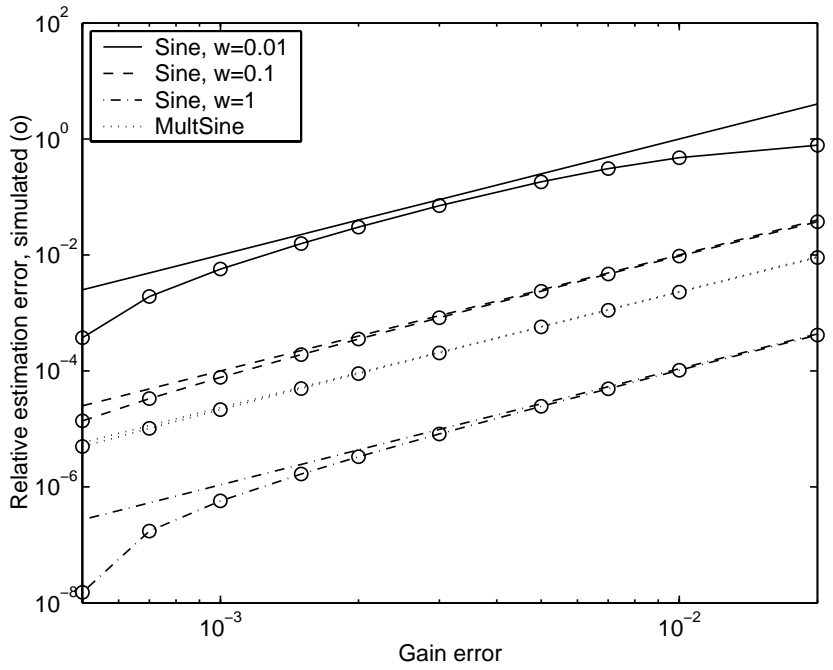

Fig. 2. Comparison between theoretical time estimation error and time estimation error from simulations as a function of gain error size, for four different input signals. The lines marked with 'o' indicate the estimation error calculated from simulations and the unmarked lines indicate the theoretical estimation errors.

\section{- Gain errors:}

Ten different gain errors between 0.0005 and 0.02 are used.

\section{- Amplitude errors:}

Ten different amplitude errors between 0.0005 and 0.02 are used.

The true time error is $0.01 T_{s}$ in all the simulations and the number of samples is $N=100000$. Figure 2 shows a comparison between theoretical estimation errors and estimation errors from simulations as a function of gain error size. Figure 3 shows a comparison between theoretical estimation errors and estimation errors from simulations as a function of amplitude error size. All these simulations confirm that the theoretically calculated value is a good approximation of the actual time estimation errors caused by amplitude and gain errors. The deviation for large errors in gain and amplitude is caused by the neglecting of higher order terms in the Taylor expansion and the deviation for small amplitude and gain errors is caused by too little data.

\section{CONCLUSION}

We have investigated a method for blind estimation of time errors in a time interleaved A/D converter. The estimation method assumes that gain and amplitude errors are removed before the estimation of time errors. We have in this paper investigated how gain and amplitude errors influence the performance of the time estimation. We have calculated approximate expressions for this influence and verified these expressions with simulations. From the calculated estimation errors, equations (19), (26) and (29), we can conclude that small gain and amplitude errors do not effect the estimation accuracy much for most input signals. However, if the input signal is very slowly varying the influence can be significant.

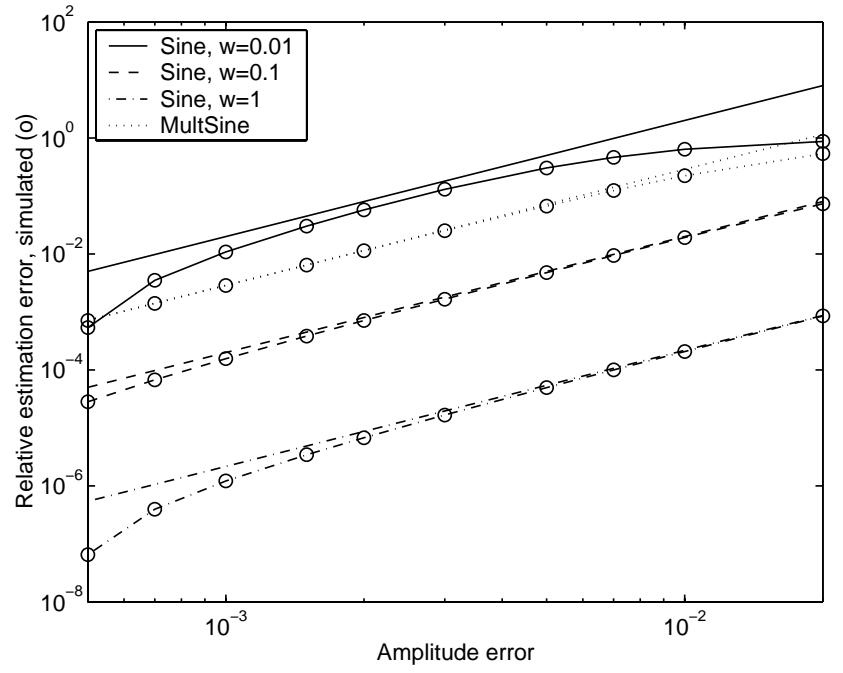

Fig. 3. Comparison between theoretical time estimation error and time estimation error from simulations as a function of amplitude error size, for four different input signals. The lines marked with 'o' indicate the estimation error calculated from simulations and the unmarked lines indicate the theoretical estimation errors.

\section{REFERENCES}

[1] Y-C Jenq, "Digital spectra of nonuniformly sampled signals: A robust sampling time offset estimation algorithm for ultra high-speed waveform digitizers using interleaving," IEEE Transactions on Instrumentation and Measurement, vol. 39, no. 1, pp. 71-75, February 1990.

[2] J.J. Corcoran, "Timing and amplitude error estimation for time-interleaved analog-to-digital converters," US Patent nr. 5,294,926, October 1992.

[3] H. Jin and E.K. Lee, "A digital-background calibration technique for minimizing timing-error effects in time-interleaved ADC's," IEEE Transactions on Cicuits and Systems, vol. 47, no. 7, pp. 603-613, July 2000.

[4] J. Elbornsson and J.-E. Eklund, "Blind estimation of timing errors in interleaved AD converters," in Proc. ICASSP 2001. IEEE, 2001, vol. 6, pp. 3913-3916.

[5] L. Ljung, System Identification, Theory for the user, PrenticeHall, 2 edition, 1999.

[6] A. Papoulis, Signal Analysis, McGraw-Hill, 1977.

[7] J. Elbornsson, Equalization of Distortion in A/D Converters, Lic. thesis 883, Department of Electrical Engineering, Linköping University, Linköping, Sweden, April 2001.

[8] G. Dahlquist Å. Björk, "Numerical mathematics,” July 1997. 